\title{
Study of Glass Fiber Textile Control Based on Image Processing and Neural Network
}

\author{
Shu-Qian Chen ${ }^{1, *}$, Yang-Lie Fu ${ }^{2}$ and Gui-Zhi Bai ${ }^{3}$
}

\author{
${ }^{I}$ School of Computer Engineer, Huaihai Institute of Technology, Lianyungang, Jiangsu, China; ${ }^{2}$ School of Computer \\ Engineer, Huaihai Institute of Technology, Lianyungang, Jiangsu, China; ${ }^{3}$ School of Computer Engineer, Huaihai \\ Institute of Technology, Lianyungang, Jiangsu, China
}

\begin{abstract}
Research on weft fiber cut problems of glass fiber has improved the efficiency of textile production. Glass fiber textile machine is a major producer machine of glass fiber cloth. In production, detection of textile machines weft usually adopts the contact type, requiring the weft to maintain a certain pressure on the sensor. This way can make the glass fiber weft fluff, and produce glass fiber dust, and may also cause harm to the human health and damage to the textile machine. Using video monitoring method detection weft, speed and image identification rate will directly affect the stability of the system. This paper presents a detection method of glass fiber textile's weft fiber cut based on neural network, selecting multiple features which are directly related to the image with the weft as neural network input vector, through repeated training samples to remove tiny ripple effects which are caused by weft textile jitter, overcome the traditional method detection accuracy is not high. Experiments show that this method can effectively avoid the weft jitter, making accurate detection of the weft fiber cut, and achieving satisfactory results.
\end{abstract}

Keywords: Weft detection, glass fiber textile, image identification, neural network.

\section{INTRODUCTION}

With the rapid development of wind power generation technology, large wind turbine installed capacity increases fast. Single capacity of the wind generator depends on the diameter of the blade. At present, the main material of the blade is glass fiber cloth [1] and carbon fiber. Glass fiber cloth has great advantage in price and cost, so its use is on large amount. Ordinary civilian textile machinery, using the contact detection method to detect the weft, does not apply to glass fiber. Because it will make the glass fiber weft fluff, causes when the shaft of rapier loom drag the weft, resistance increased, change the motion path of the shaft, cut the warp and damage to the textile machine often occurs. And the glass fiber dust which is generated by the contact weft detector has a huge damage to human health. So using noncontact video detection is a better solution. However, in practical applications the scheme is limited by image identification speed, accuracy and the processor speed of the textile machine's controller. In order to speed the process, using high-speed ARM processor and dedicated DSP chip, achieved better detection. In this paper we discussed the weft detection method from the perspective of image identification algorithms, the specific implementation on DSP processor. This paper does not involve the ARM and DSP control process.

The traditional weft image detection method based on gray variance made detection of the weft fiber cut through the dependency between image gray variance and normal

\footnotetext{
*Address correspondence to this author at the School of Computer Engineer, Huaihai Institute of Technology, Lianyungang, Jiangsu, China;

Tel: +86051885895390; Fax: +86051885895386; Email: chenshq@126.com
}

weft, which is by analyzing the extraction Textile process of weft image, and control of textile machines in real time. However, when the weft is traction, with the shaft of the action, will cause the weft pulsation and the weft image gray variance changed along with it [2], at this time the traditional detection systems based on gray variance will be mistaken for a weft fiber cut occurs, then make the appropriate control action, affecting the textile jobs. This shows that the tradition weft detection method based on gray variance cannot be avoided caused by shaft movement, resulting in detection accuracy is not high, can not meet the weft fiber cut accuracy requirements.

According to the traditional detection method based on gray variance jitter caused by the weft fiber cut detection accuracy is not high. This paper presents a detection methods of glass fiber textile's weft fiber cut based on neural network-based, select multiple features which are directly related to the image with the weft as neural network input vector, through repeated training samples to remove tiny ripple effects which are caused by weft textile jitter, overcome the traditional method detection accuracy is not high.

\section{TEXTILE MACHINE WEFT DETECTION PRINCI- PLE}

Weft fiber cut detection is the key technology of glass fiber textile machines safe, efficient production and ensure the health of workers. However, due to the condition of noncontact detection, we are unable to use observed detection to Textile weft, which was once the difficult problem plaguing researchers. With the development of information technology, researchers began to apply image processing techniques in the areas of textiles. Because this technique does not re- 
quire weft for the contact detection, but through the acquisition of weft textile image, and processing and analysis the image to determine the weft fiber cut, successfully resolved the problem which has plagued researchers. Using digital image processing method for weft fiber cut detection can be divided into four parts: image acquisition unit, image preprocessing unit, fiber cut detection unit, the response unit. Detection principle block diagram is shown in (Fig. 1).

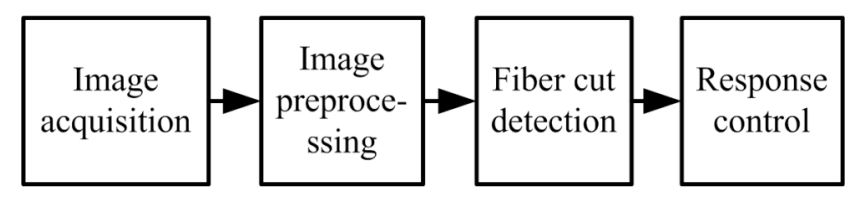

Fig. (1). Principle block diagram.

Known by the principle block diagram, the glass fiber textile machine detection step is, first using camera equipment to obtain textile machine's weft images, then make preprocessing on the images such as de-noising, image segmentation, remove image noise, extract multiple image feature vector, construction of corresponding relationship between the image features and weft works, according to the state of the image feature vector detection weft fiber cut, finally respond to adjustment unit's response to the test results. Among them, the most important part is the fiber cut detection unit, that is choose which image characteristics to construct the direct contact with fiber cut, in order to ensure the accuracy detection of fiber cut. Weft normal working image is shown in (Fig. 2) [3].

But the traditional weft image detection method based on gray variance is just use the gray of the image to establish the contact with fiber cut. To the steady weft textile, its image gray change smaller, distribution will be more uniform, therefore, the variance of image gray is smaller, the textile machine weft is more normal at this time. Gray variance characteristic vector calculation formula is:

$$
\sigma^{2}=\sum_{i=1}^{m} \sum_{j=1}^{n}(g(i, j)-a v)^{2}
$$

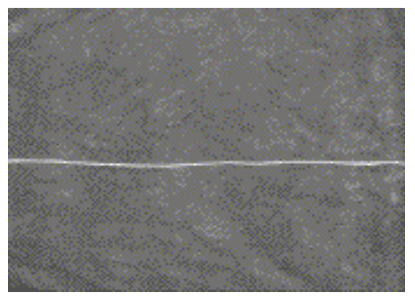

(a) Reference image

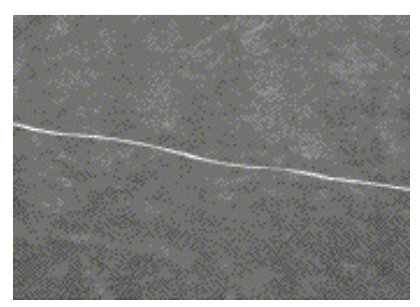

(c) Downward tilt chart

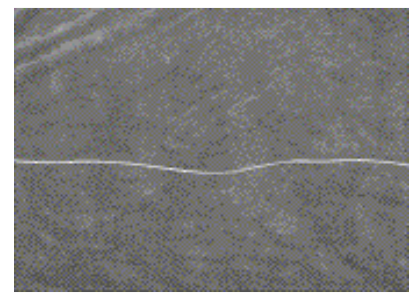

(b) Wave Chart

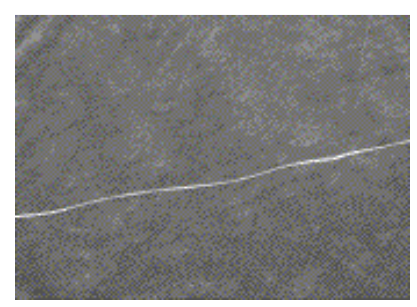

(d) Upward tilt chart
Fig. (2). Weft work image.
In the formula: $g(i, j)$ is image pixel gray value, $a v$ is the average value of all gray values in the image. Discrimination function of weft fiber cut detection based on gray variance is

$$
\begin{cases}\sigma^{2}>\lambda & \text { Weft cut, control action } \\ \sigma^{2} \leq \lambda & \text { normal }\end{cases}
$$

In the formula: $\lambda$ is detection threshold, the general value is 0.5.However, when the textile machine works, drives the weft occurs pulsation, the weft image gray variance changed along with it, at this time, the value of $\sigma^{2}$ which is obtained according to detection method based on gray variance become larger, therefore, the system will mistakenly believe that this time occurs weft fiber cut, and make the control unit moves, resulting in textile machine downtime issues. The calculation formula of fiber cut detection accuracy is:

$$
w=\frac{\left(P(t)-P^{\prime}(t)\right) / t}{P(t)} \times 100 \%
$$

In the formula: $\mathrm{w}$ is stability detection accuracy, $P(t)$ is the number of obtain weft image in unit time, $P(t)$ is the number of error detection image in unit time. The traditional weft image detection method based on gray variance, because it only relies on image gray variance to detect the accuracy, can not effectively remove pulsation effects of weft jitter, causing a large number of image error detection, from formula 3 can know that its detection accuracy is very low and does not meet the accuracy requirements.

\section{WEFT DETECTION METHOD BASE ON NEURAL NETWORK}

In recent years, Neural Network has aroused more and more interest and universal attention. Image identification is carries on various transformation and transformation of the actual image, achieve the purpose of identification. Typically, image information is constituted by the twodimensional spatial information, which has very large amount of information, before identification often require need signal impedance matching, amplitude adjustment, digital filtering, segmentation and so on. Then use various algorithms for identification. The neural network is introduced in image identification, making the video surveillance system with self-learning function. So as to solve these problems: the complex monitoring environmental information, background knowledge is not clear, inference rules are not clearly, allow the image has a larger defect, distortion. With the advantage, the algorithm is faster, adaptive performance is good, higher resolution. Therefore the monitoring system composed of neural network has good regulation ability and robustness [4-6], and able to adopt the variation of the weft t monitoring image.

\subsection{Image Preprocessing}

Although CCD camera can get high quality, high resolution image, however, due to the influence of the external environment image may have many noise, if make the direct detection and analysis on the acquired images, will unable to get accurate test results, so prior to the detection and analysis, need to make preprocessing to the image, such as elimination of noise and so on, in order to improve image quality. 


\section{(1) Histogram Weighted Average}

Image gray histogram reflects the various gray scale of image and the relationship of the gray scale appears number in the image. Making Weight average operations to the histogram of image can effectively filter out the high-pass noise in the image, specific algorithm formula is:

$$
\operatorname{AVER}(i, j)=\frac{\sum_{(s, t) \in S_{m^{*+n}}} p(i+s, j+t) g(i+s, j+t)}{\sum_{(s, t) \in S_{m_{n}^{*}}} p(i+s, j+t)}
$$

In the formula: $g(i, j)$ is image pixel gray value, $p(i, j)$ is corresponds to the weight of image pixels, $(s, t) \in S_{m \times n}$ is belong to all the pixels in the image.

\section{(2) Mean Filter}

After histogram equalization to the image, high-pass noise of the image is filtered out, but low-pass noise is still exists, affecting the quality of the image. Continue the mean filter on the image, to filter out low-pass noise. Suppose the image after making gray histogram weighted average is $\mathrm{g}$, the image continue to mean the filtered is $\mathrm{f}$, the size of the entire image is $M \times N$, the size of filter area is $m \times n$. Filter formula is:

$$
\begin{array}{r}
f(i, j)=\frac{\sum_{i} \sum_{j}(a b s(g(i, j)-g(i, j+1)))}{K} \\
+\frac{\sum_{i} \sum_{j}(a b s(g(i, j)-g(i+1, j)))}{K}
\end{array}
$$

In the formula: $\mathrm{K}$ is the total number of pixels which are made the difference operator, that is $K=(M-1) \times(N-1)$. Through image preprocessing, image noise removal, get the high quality image.

\subsection{Fiber Cut Detection}

As the weft textile machine work to bring the impact of jitter, jitter has the characteristics of pulsation and very complex. Using CCD camera to obtain images, after de-noising preprocessing of the image, the image will still leave large disturbances, so we need select multiple image feature vector, which are directly related to weft jitter, to characterization of the weft cut phenomenon. The detection algorithm of glass fiber textile's weft fiber cut based on neural network is selected eight feature vector which are directly relevant to weft cut, image center, image fiber area ratio, Fiber height, Fiber Centre offset distance, Fiber Centre offset distance variance, Image average gray, the average gray variance , texture feature entropy and use them as neural network input feature vectors.

(1) Image center $C(x, y) . \mathrm{X}$-axis coordinate of the fiber center is the cut-point average of fiber around the edge of the $y$-axis direction. Similarly y-axis coordinate of the fiber center is the cut-point average of fiber around the edge of the $\mathrm{x}$ axis direction

$$
C_{x}(x, y)=\frac{1}{2}(a+b), C_{y}(x, y)=\frac{1}{2}(h+d)
$$

(2) Image fiber area $S_{h t}$ and fiber area ration. Set a $M \times N$ pixel fiber image, assuming that the area of each pixel is s, the entire fiber area is $S_{\text {total }}=M \times N \times s$, on the image segmentation, removing the background, assumed glass fiber area is $S_{h t}$, the total number of pixels is $\theta, S_{h t}=\theta \times s$, glass fiber area ratio $\eta_{\eta=\frac{S_{h t}}{S_{\text {tout }}}}=\frac{\theta}{M \times N}$.

(3) Fiber height $H(x, y)$.In fiber image, the pixels above a certain threshold as the fiber image, the intersection of the fiber along the $\mathrm{x}$ axis tangent and vertex is height corresponding to the coordinates, that is

$$
H_{y}(x, y)=\max \left(h\left(x, y_{1}\right), h\left(x, y_{2}\right), \cdots h\left(x, y_{n}\right)\right)
$$

In the formula: $H_{x}(x, y)$ is $H_{y}(x, y)$ corresponding to the coordinates of the $\mathrm{x}$-axis direction, $\mathrm{n}$ is the number of all glass fiber pixels.

(4) Fiber Centre offset distance $D$. Fiber Centre offset distance is the distance from the effective fiber center to image center.

$$
D=\sqrt{\left(X-X_{0}\right)^{2}+\left(Y-Y_{0}\right)^{2}}
$$

In the formula, $\left(X_{0}, Y_{0}\right)$ is image center coordinate, fiber center coordinate is $(X, Y)$, its value is calculated by formula (6):

$$
X=\frac{\sum_{y=1}^{N} \sum_{x=1}^{M} x g^{\prime}(x, y)}{\sum_{y=1}^{N} \sum_{x=1}^{M} g^{\prime}(x, y)}, Y=\frac{\sum_{x=1}^{M} \sum_{y=1}^{N} y g^{\prime}(x, y)}{\sum_{x=1}^{M} \sum_{y=1}^{N} g^{\prime}(x, y)}
$$

In the formula, $g^{\prime}(x, y)$ is calculated by formula (10):

$$
g^{\prime}(x, y)=\left\{\begin{array}{l}
g(x, y), g(x, y) \geq g_{a v} \\
0, g(x, y) \leq g_{a v}
\end{array}\right.
$$

In the formula, $g(x, y)$ is the gray of glass fiber image coordinates $(x, y), g_{a v}$ is average gray.

(5) Fiber Centre offset distance variance $\delta_{D}$. Fiber Centre offset distance variance reflects the intensity of swing glass fiber in the center of the image, also reflects the important feature of fiber breakage. Make fiber center offset distance variance of Collection of $\mathrm{k}$ images in $\mathrm{t}$ time as the fiber image feature vector, Fiber Centre offset distance variance $\delta_{D}$ is defined as:

$$
\delta_{D}=\sqrt{\frac{1}{K} \sum_{j=1}^{K}\left(\bar{D}-D_{j}\right)^{2}}
$$

(6) Image average gray. The average gray of glass fiber image can be used to characterize whether there is breakage of glass fiber. After fiber breakage, fiber will naturally fall from the effects of gravity, disappear or partially disappear in the image. In practical applications, according to the difference between the fiber and the background, set a threshold, average gray is greater than this threshold, we think that fiber works fine, otherwise think that the fibers have broken 
filaments. For a glass fiber image, its average value of gray can be expressed as:

$$
g_{a v}=\frac{1}{M N} \sum_{i=1}^{M N} g_{i}
$$

In the formula, $g_{i}$ is the gray of pixel $\mathrm{i}$ in the image, $\mathrm{M}$ and $\mathrm{N}$ are respectively the pixel row and column of the image. Since there are all kinds of noise and fiber jitter on site, in order to filter out the interference, can acquire multiple images in the practical application, the average gray value is:

$$
\overline{g_{a v}}=\frac{1}{K} \sum_{j=1}^{K} g_{a v j}
$$

In the formula, $\mathrm{K}$ is the number of images collected in the continuous time $\mathrm{t} ; g_{a v j}$ is the average gray of image $\mathrm{j}$, $\overline{g_{a v}}$ is the average gray of $\mathrm{K}$ image within the time t.

(7) The average gray variance. In extracting features of glass fiber image, we introduced the image average gray variance which can reflect the state of the real glass fibers. In time $\mathrm{t}$, average gray level variance of $\mathrm{k}$ image is defined as:

$$
\delta_{g}=\sqrt{\frac{1}{K} \sum_{j=1}^{K}\left(\overline{g_{a v}}-g_{a v j}\right)^{2}}
$$

(8) Texture feature entropy. Use the poor co-occurrence matrix based on the regional average binary gray difference texture feature extraction algorithm, the algorithm steps are shown in (Fig. 3),

Defining area binary gray, the window size increases will make computing dramatically increases, usually use the $3 * 3$ window to characterize all the pixel gray value of the region, the formula is as follows:

$$
G=\left(g_{c}, g_{0}, g_{1}, \cdots, g_{7}\right)
$$

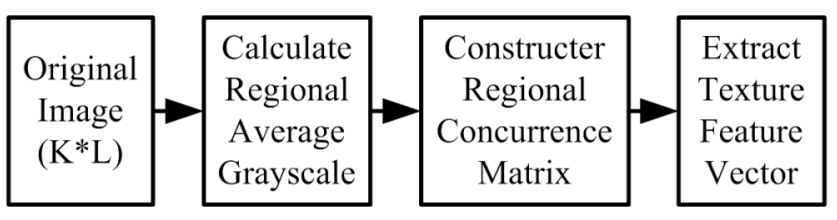

Fig. (3). Step of Regional average binary gray difference cooccurrence matrix texture feature extraction.

In the formula, $g_{c}$ is the center pixel gray value of the region, $g_{i}$ is eight adjacent points pixel gray values of the region. The difference between the neighborhood points pixel gray value and the center point gray value can use the following formula markup:

$$
\begin{aligned}
& \left(g_{i}-g_{c}\right)= \begin{cases}1 & \left(g_{\mathrm{i}}-g_{c}\right)>0 \\
0 & \left(g_{\mathrm{i}}-g_{c}\right) \leq 0\end{cases} \\
& \left(g_{i}-g_{c}\right)= \begin{cases}1 & \left(g_{\mathrm{i}}-g_{c}\right)<0 \\
0 & \left(g_{\mathrm{i}}-g_{c}\right) \geq 0\end{cases}
\end{aligned}
$$

We can obtain two 8-bit binary sequence of the region bright and dark texture distribution. According to the right of center pixel level direction rotates anticlockwise, greater sequence is given the weight $2^{p}(p=0,1, \cdots 7)$, less sequence is given the weight $2^{q}(q=7,6, \cdots 0)$ and the range of practical value is discrete values in $[0,255]$. These two values indicated texture distribution of the neighborhood pixel which is greater than or less than the center pixel gray values. Suppose an extract regional average binary gray difference texture image size is $M \times N$, Starting from this area that average binary gray difference is $i$, the position coordinates is $(x, y)$, statistics the number $P(i, j, d, \theta)$ of regional appear at the same time., which distance of it is $d$,direction is $\theta$, regional average binary gray difference is $j$, position coordinates is $(x+\Delta x, y+\Delta y)$. Can be expressed by the formula:

$$
P(i, j, d, \theta)=\{[x, y],[x+\Delta x, y+\Delta y] \mid f(x, y)=i, f(x+\Delta x, y+\Delta y)=j\}
$$

In the formula, $i, j$ is regional average binary gray difference, the range is $[-255 / 8,255 / 8]$.

$(x, y)$ is the position coordinates of the region with different primitives mode value, $x=0,1 \cdots M, y=0,1 \cdots N$.

$\theta$ is the direction of regional co-occurrence matrix generated, take the $0^{\circ}, 45^{\circ}, 90^{\circ}$ and $135^{\circ}$ four directions constructed regional co-occurrence matrix. The texture feature Entropy calculation formula is as follows:

$$
f=-\sum_{i=-32}^{32} \sum_{j=-32}^{32} p(i, j) \log _{2} p(i, j)
$$

\subsection{Fiber Cut Detection}

First, build a neural network model, its construction formula is:

$$
\begin{aligned}
& y_{j}=\sigma\left[\sum_{k=1}^{K} w_{k j} \sum_{i=1}^{I} x_{i} \psi_{a, b}\left(\frac{i-b_{k}}{a_{k}}\right)\right] \\
& \psi_{a, b}(x)=\cos (1.75 x) \exp \left(-x^{2} / 2\right) \\
& \sigma(x)=1 /(1+\exp (x))
\end{aligned}
$$

In the formula: $I, K, J$ represent the neural network input layer nodes number, network nodes number of Hidden layer, network nodes number of output layer [7]. ${ }^{w_{k j}}$ is the connection weights of network output layer node $j$ and the hidden layer node $k$.

Then, iterative search make to the neural network, the search formula is:

$$
\begin{aligned}
& v_{i d}(t+1)=w(t) v_{i d}(t)+c_{1} r_{1}\left(p_{i d}(t)-x_{i d}(t)\right) \\
& x_{i d}(t+1)=x_{i d}(t)+v_{i d}(t+1)
\end{aligned}
$$

In the formula: $c_{1}=c_{2}=1.4994$ is search speed constant, $r_{1}$ and $r_{2}$ are random values in the range 0 to 1 . In order to avoid iteration into the local minimum, need for a directed mutation operation. Its directed mutation formula is:

$$
\begin{aligned}
& v_{i d}=\operatorname{rand} \times v_{\text {max }}(d) \\
& p_{i d}=x_{i d}
\end{aligned}
$$

In the formula: rand is a random value in the range 0 to 1 , The maximum speed of iteration is denoted by $v_{\max }$. 
Using $v_{i d}$ which is obtained of the final search iteration determine the weft cut, discrimination function is:

$$
\left\{\begin{array}{cl}
v_{i d}>\lambda & \text { Weft cut, control action } \\
v_{i d} \leq \lambda & \text { normal }
\end{array}\right.
$$

Where $\lambda$ is the discrimination threshold ${ }^{[8,9]}$, the general value is 0.5 .

In this way, using eight characteristics vector which are directly related to the weft jitter, construction neural network using search iterations, avoiding rely on only one gray variance characteristic vectors cannot effectively remove weft jitter pulse interference which is caused by textile machines working, can accurately detect weft fiber cut, to meet the glass fiber textile machine's requirements of fiber cut detection accuracy.

Glass fiber textile Control Based on Neural Network's control algorithm flowchart is shown in (Fig. 4):

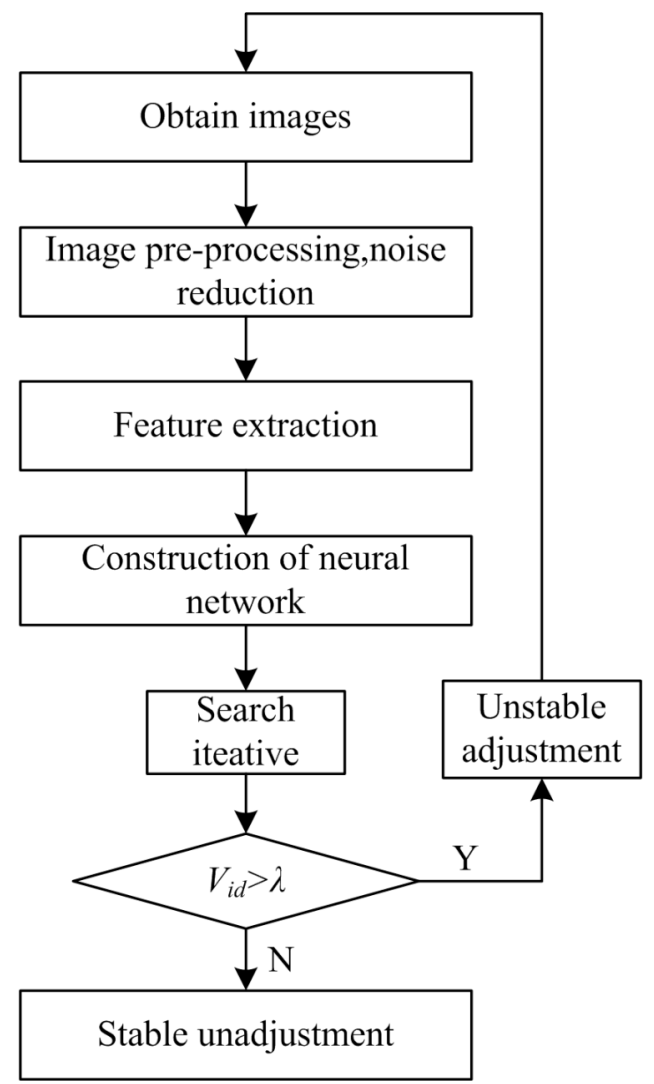

Fig. (4). Neural network detection algorithm processes.

\section{THE RESULTS OF IMAGE IDENTIFICATION}

The algorithm applied to the glass fiber textile weft video monitoring system, From the CCD surveillance video extract some image, extracting its feature vectors, train BP neural network. After training, employ the simulation test set to test the neural network.

By compared Figs. (5) and (6) can be seen, under the same number of detections, for the identification mean square error of the monitor image, BP neural network algorithm is an order of magnitude lower than the Gray variance algorithm, and the correct rate of identification is improved about $16 \%$, Shown in (Table 1).

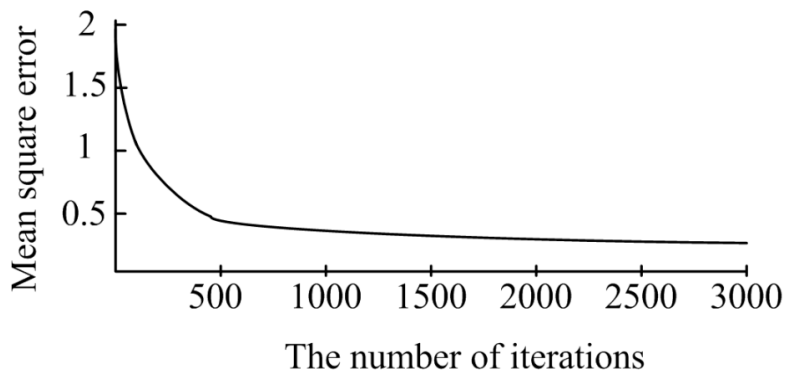

Fig. (5). Gray variance algorithm in image identification error.

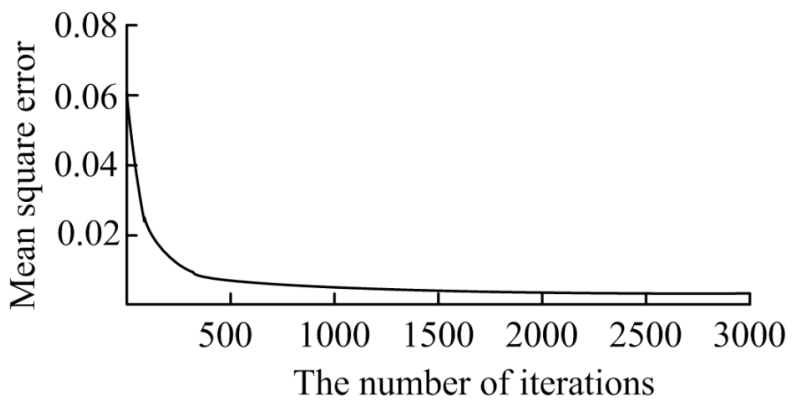

Fig. (6). BP Network algorithm in image identification error.

Table 1. Algorithm Efficiency Comparison Table

\begin{tabular}{|c|c|c|}
\hline Algorithm & Error detection image & Identification rate \% \\
\hline \hline $\begin{array}{c}\text { BP Network } \\
\text { algorithm }\end{array}$ & 5 & 96.88 \\
\hline $\begin{array}{c}\text { Gray variance } \\
\text { algorithm }\end{array}$ & 27 & 83.13 \\
\hline
\end{tabular}

Thus it can be seen, the proposed algorithm can improve the convergence speed without increasing the complexity of algorithm, and do not need to establish a precise mathematical model of recognition object, has strong anti-jamming capability, network rapid convergence speed. This indicates that BP network algorithms make it slower convergence of the algorithm, easy to fall into local minimum problem showing great advantages.

\section{APPLICATIONS CONCLUSIONS}

The traditional weft image detection method based on gray variance cannot effectively remove the outside interference, under the influence of pulsation which is cause by weft jitter of textile machines work, prone to error detection. In experiment error detection image number is 27 , resulting in weft fiber cut detection accuracy is very low, only $83.13 \%$, the detection methods of glass fiber textile's weft fiber cut based on neural network which is presented in this paper, not rely on a single gray variance characteristic vec- 
tor, but selected eight feature vector which is directly relevant to weft cut for weft fiber cut detection. Experimental data shows, the number of error detection image is only 5, which is far less than traditional methods, eventually the detection accuracy is $96.88 \%$, obviously superior to the traditional method.

The experimental results also show that, the image recognition method based on neural network is effective, feasible. With the development of computer technology and artificial intelligence theory, the image identification technology will have broad development and application in the field of video monitoring, image identification, intelligent instrumentation, robot vision and other fields.

\section{CONFLICT OF INTEREST}

The author(s) confirm that this article content has no conflicts of interest.

\section{ACKNOWLEDGEMENT}

Declared none.

\section{REFERENCES}

[1] M.A. Hui, W. Fei and C.U.I Wei, “Application of combustion diagnosis and operation instruction system in 300 MW utility boiler", Electric. Power, vol. 37, no. 9, pp. 30-33, 2004.

[2] Y. Wan-ye, C.Guang-hua and L. Chuan-wei, "Design and development of flame image monitoring systems of power plant boilers", Electric. Power Sci. Eng., vol. 1, pp. 48-50, 2006.

[3] Y. Yan, G.Lu and M.colechin, "Monitoring and characterization of pulverized coal flames using digital imaging techniques", Fuel, vol 8, no.1, 647-656, 2002.

[4] X. Bao-chang, Z. Ding-yuan and C. Liang,"Research on combustion stability based on flame images", Comput. Eng. Appl., vol. 3, no. $24,2011$.

[5] D. Xi-mei, Y. Lei, C. Hao and W. Wei-jia, " Study on algorithm of flame detection based on image processing", J. Logistic. Eng. Univ., vol. 27, no.2, pp. 90-95, 2011.

[6] Z. Chun-guang, Z. Huai-chun and L. Chun, "Diagnosis and analysis of combustion stability based on flame images", Hubei Electric Power, vol. 18, no.3, pp. 210-217, 2006.

[7] C. Shu-qian, Z.Li-hong, "Optimization Tuning of PID Parameters about PID Controller Based on BP Neural Network", Comput. Simul., vol. 27, no.10, pp. 171-174, 2010.

(C) Chen et al.; Licensee Bentham Open.

This is an open access article licensed under the terms of the Creative Commons Attribution Non-Commercial License (http://creativecommons.org/licenses/by-nc/3.0/) which permits unrestricted, non-commercial use, distribution and reproduction in any medium, provided the work is properly cited. 